\title{
RESEARCH
}

Open Access

\section{FBXL6 governs c-MYC to promote hepatocellular carcinoma through ubiquitination and stabilization of HSP90AA1}

Weidong Shi ${ }^{1,2,3}$, Lanyun Feng ${ }^{1,2,3}$, Shu Dong ${ }^{1,2,3}$, Zhouyu Ning ${ }^{1,2,3}$, Yongqiang Hua ${ }^{1,2,3}$, Luming Liu ${ }^{1,2,3}$, Zhen Chen ${ }^{1,2,3}$ and Zhiqiang Meng ${ }^{1,2,3^{*}}$

\begin{abstract}
Background: Heat shot protein 90 (HSP90) AA1 functions as an onco-protein to regulate the assembly, manipulation, folding and degradation of its client proteins, including c-MYC. However, little is known about the mechanism of HSP90AA1 regulation.

Methods: Transcriptome RNA-sequencing data of hepatocellular carcinoma (HCC) samples were used to detect the mRNA expression of FBXL6. Immunoprecipitation/Mass Spectrum (IP/MS) method was used to identify the interacting proteins of FBXL6. The co-immunoprecipitation assay was used to determine the interaction between FBXL6 and HSP90AA1. The in vivo ubiquitination assay was performed to determine the regulation of HSP90AA1 by FBXL6. Luciferase reporter and chromatin immunoprecipitation (ChIP) assays were used to determine the transcriptional regulation of FBXL6 by c-MYC. Immunohistochemical (IHC) staining was performed to study the correlation of FBXL6 and HSP90AA1 protein expression in $87 \mathrm{HCC}$ samples. Cell counting and colony formation assays were implemented to detect the biological effects of FBXL6 on the growth of HCC cells in vitro. The effect of FBXL6 on HCC tumor growth in vivo was studied in a tumor xenograft model in mice.
\end{abstract}

Results: Here, we identified the orphan F-box protein FBXL6, a substrate recognition subunit of an SCF (Skp1-Cul1-Fbox protein) complex, as the ubiquitin ligase for HSP90AA1. FBXL6 promoted K63-dependent ubiquitination of HSP90AA1 to stabilize it. Through analysis of the TCGA dataset, we found that FBXL6 was significantly increased in HCC tissues and positively correlated with c-MYC pathway. FBXL6 accumulation in HCC causes the stabilization and activation of C-MYC by preventing HSP90AA1 degradation. The activated c-MYC directly binds to the promoter region of FBXL6 to induce its mRNA expression.

\footnotetext{
* Correspondence: mengzhq@yeah.net

'Department of Integrative Oncology, Fudan University Shanghai Cancer Center, 270 Dong An Road, Shanghai 200032, China

${ }^{2}$ Department of Oncology, Shanghai Medical College, Fudan University, Shanghai, China

Full list of author information is available at the end of the article
}

(C) The Author(s). 2020 Open Access This article is licensed under a Creative Commons Attribution 4.0 International License, which permits use, sharing, adaptation, distribution and reproduction in any medium or format, as long as you give appropriate credit to the original author(s) and the source, provide a link to the Creative Commons licence, and indicate if changes were made. The images or other third party material in this article are included in the article's Creative Commons licence, unless indicated otherwise in a credit line to the material. If material is not included in the article's Creative Commons licence and your intended use is not permitted by statutory regulation or exceeds the permitted use, you will need to obtain permission directly from the copyright holder. To view a copy of this licence, visit http://creativecommons.org/licenses/by/4.0/ The Creative Commons Public Domain Dedication waiver (http://creativecommons.org/publicdomain/zero/1.0/) applies to the data made available in this article, unless otherwise stated in a credit line to the data. 
(Continued from previous page)

Conclusion: Collectively, our data revealed an unknown FBXL6-HSP90AA1-c-MYC axis which might contribute to the oncogenesis of HCC, and we propose that inhibition of FBXL6 might represent an effective therapeutic strategy for HCC treatment.

Keywords: Hepatocellular carcinoma, Heat shock proteins, HSP90AA1, SCF complex, C-MYC, FBXL6, Ubiquitination, Fbox protein, E3 ligase, Degradation

\section{Background}

Hepatocellular carcinoma $(\mathrm{HCC})$ is one of the most common cancer in the world and the second malignant tumor of global cancer mortality, and its morbidity and mortality are gradually increasing [1]. The pathogenesis of HCC is extremely complicate and the discovery of new molecular drug targets will benefit HCC treatment [2].

The ubiquitin (Ub)-proteasome system (UPS) plays a prominent role in a variety of cellular activities, including cell cycle control, apoptosis, DNA damage repair, immune response and tumorigenesis [3]. Ubiquitination is catalyzed by a three-enzyme cascade consisting of the E1 Ub-activating enzyme, the E2 Ub-conjugating enzyme, and the E3 Ub-protein ligase [4]. In UPS, Ub modifies protein substrates mostly in the form of a K48or K11-linked polyUb chain, which serves as a signal for proteasome-dependent degradation [5]. However, K63linked polyUb chain is not associated with proteasome degradation of the substrate protein [6].

The selectivity of Ub-mediated proteolysis is determined by the E3 ligases which could be grouped into two classes based on their structural features: the RING (really interesting new gene) E3s and the HECT (homologous to the E6AP carboxyl terminus) E3s. The RING E3s constitute the largest E3 ligases family with more than 600 documented members, which directly catalyze the transfer of ubiquitin from an E2 to a substrate [7]. The substrates-recruiting and catalytic modules could be found in a single polypeptide or in different subunits of a E3 complex, such as the anaphase-promoting complex (APC) and the Cullin-RING ligases (CRLs) [8]. In mammals, there are approximately 200 CRLs has been reported. Each of CRLs contains a different Cullin subunit that using its carboxyl terminus to bind to the E2 enzyme and $\mathrm{N}$ terminus to bind to the substrate recognition factors. The CRL1 ligases, better known the SCF (Skp1-Cul1-F-box protein) complex, are the best characterized. SCF is a four-protein complex consisting of the constant Cullin1, RBX1, SKP1 and one of $\sim 70$ various F-box proteins [9]. Early studies have demonstrated that F-box proteins play indispensable roles in cell cycle regulation [10-12], and in recent years more and more F-box proteins have been reported to be closely related to tumorigenesis $[13,14]$. However, given the larger number of F-box proteins, only a few F-box proteins have identified substrates and functions.

Heat shock proteins (HSPs) are a class of highly conserved proteins during biological evolution and widely found in prokaryotic and eukaryotic organisms [15]. HSPs could be induced under diverse stress conditions (virus infection, hypoxia, ultraviolet radiation, etc.). HSPs are both biomarkers of cellular stress response and also important molecular chaperone proteins in cells [15]. HSPs participate in maintaining the correct folding of the client's protein, enabling the protein to form the conformation required for physiological functions, thereby playing an important role in regulating the balance of protein synthesis/degradation and protein localization [16]. HSPs are mainly divided into five families: HSP90 family (83-90 kD), HSP70 family (66-78 kD), HSP60 family, small molecule smHSP family (15-30 kD), and macromolecular HSPs with molecular weights ranging from 100 to $110 \mathrm{kD}$. Among them, HSP90 is abundant in cells, accounting for 1 to $2 \%$ of total cellular protein [17]. In humans, there are four Hsp90 isoforms including the cytoplasmic Hsp90 $\alpha$ and Hsp90 $\beta$, as well as the endoplasmic reticulum isoform Grp94 and mitochondrial isoform TRAP1, respectively [18]. Hsp90 $\alpha$ (Hsp90AA1), encoded by the HSP90aa1 gene, is composed of three major domains: the $\mathrm{N}$-terminal domain, the intermediate domain, and the C-terminal domain. These three domains work together to play the molecular chaperone function of HSP90AA1, which is dependent on the binding of ATP to the ATPase domain at the N-terminus [19]. The binding and hydrolysis of ATP produces a conformational transition that regulates the assembly of the multi-subunit complexes involved. HSP90AA1 plays an important role in the assembly, manipulation, folding and degradation of its client proteins. Numerous studies have shown that inhibition of HSP90AA1 function can lead to degradation of its client protein through the ubiquitin-protease pathway [20]. Many of the client proteins regulated by HSP90AA1 are proto-oncogene products (such as c-MYC) or important signal transduction factors during tumor pathogenesis, which are closely related to the occurrence and development of tumors [21, 22]. Thus, inhibition of HSP90AA1 might affect cancer cells growth and survival from multiple pathways, making HSP90AA1 a promising antitumor drug target. 
Here, through analysis of the TCGA dataset, we found that the mRNA expression of an orphan F-box protein FBXL6 was significantly increased in HCC compared with normal tissues and positively correlated with cMYC expression. We further showed that FBXL6 forms a classical SCF E3 ligase complex to exert its oncogenic roles by stabilizing HSP90AA1 to activate c-MYC, which in turn directly bound to the promoter region of FBXL6 to induce its expression. Thus, our data revealed an unknown positive feedback axis of FBXL6-HSP90AA1-cMYC, whose abnormal activation might contribute to the oncogenesis of HCC.

\section{Material and methods}

\section{Clinical samples and data acquisition}

Transcriptome RNA-sequencing data of hepatocellular carcinoma (HCC) samples were downloaded from the TCGA data portal (https://cancergenome.nih.gov/), which contained data from 374 primary HCC and 50 non-tumor tissues. Raw count data was downloaded for further analyses. To selected genes involved in the onset of HCC, differentially expressed genes between HCC and non-tumor tissues were screened via the R software Linear Models for Microarray and RNA-Seq Data (Limma) package (http:// bioconductor.org/packages/Limma/). We performed differential gene analysis of all transcriptional data, setting a $\log 2 \mid$ fold change $\mid>1$ and a false discovery rate $($ FDR $)<$ 0.05 as the cutoff values. The Wilcox-test was used for analyses.

\section{Immunohistochemical (IHC) staining}

The HCC cancer tissues and matched adjacent tissues (at least $2 \mathrm{~cm}$ from the surgical incision) were collected from 87 patients with hepatocellular carcinoma who were surgically resected at Fudan University Shanghai Cancer Center from January 2016 to December 2018. All specimens were confirmed by pathological diagnosis. No patients received radiotherapy or chemotherapy before surgery. These tissues were placed in liquid nitrogen and then transported to $-80^{\circ} \mathrm{C}$ refrigerator for storage. Written informed consent was obtained from each patient before sample collection, and the study protocol was approved by the Medical Ethics Committee of Fudan University Shanghai Cancer Center. These 87 HCC clinical samples were fixed in $4 \%$ paraformaldehyde (PFA), embedded in paraffin, sectioned and stained with haematoxylin and eosin. IHC staining of the paraffinembedded tumor tissues was performed using antiFBXL6 and anti-HSP90AA1 antibodies. We have added these information in the revised manuscript.

\section{Cell culture and reagents}

HEK293T cells and hepatocellular carcinoma cell lines SMMC-7721 and Hep3B cells were purchased from American Type Culture Collection (ATCC). Cells were cultured in Dulbecco's modified Eagle's medium (DMEM) (Invitrogen), supplemented with 10\% FBS (Gibco), 100 units $/ \mathrm{mL}$ penicillin, and $100 \mathrm{mg} / \mathrm{mL}$ streptomycin (Gibco). MG132 and Cycloheximide (CHX) were purchased from Sigma.

\section{Plasmids}

F-box protein genes were amplified from $293 \mathrm{~T}$ or SMMC-7721 cells by polymerase chain reaction and cloned into pbabe-Flag vector. pCherry.90 alpha was a gift from Didier Picard (Addgene plasmid \# 108222; http://n2t.net/addgene:108222; RRID:Addgene_108,222). c-myc-PT3EF1a was a gift from Xin Chen (Addgene plasmid \# 92046; http://n2t.net/addgene:92046; RRID: Addgene_92,046). pRK5-HA-Ubiquitin-K63 was a gift from Ted Dawson (Addgene plasmid \# 17606; http://n2 t.net/addgene:17606; RRID:Addgene_17,606). All plasmids were completely sequenced and transfected into cells by using Lipofectamine 2000 (Invitrogen) according to manufacturer's instructions.

\section{RNA interference, RNA isolation and real-time PCR}

The Lentiviral Human FBXL6 shRNA was purchased from Merck and the target sequences for short hairpin RNA (sh-RNA)-expressing plasmids were the following: FBXL6-shRNA1: CACCGGCATCAACCGTAATAG; FB XL6-shRNA2: TGGAGTGGCTTATGCCCAATC. Total RNA of cell lysate was extracted by using TRIzol reagent (Invitrogen, Shanghai). Oligo dT was used to prime cDNA synthesis. Real-time PCR was then performed by using a SYBR Green Premix Ex Taq (TaKaRa) on Light Cycler480 (Roche, Switzerland). GAPDH was used as internal control. Differences in gene expression were calculated using 2- $\Delta \Delta \mathrm{Ct}$ method. Primers used for qPCR analysis were list as follows: FBXL6 forward, 5'- GGAGACCGCATTCC CTTGG-3'; reverse, 5' - AAAACCGATTGGGCATAA GCC-3'. HSP90AA1 forward, 5' - AGGAGGTTGAGACG TTCGC-3'; reverse, 5' - AGAGTTCGATCTTGTTTG TTCGG-3'. GAPDH forward, 5' - TGTGGGCATCAATG GATTTGG - 3'; reverse, $5^{\prime}$ - ACACCATGTATTCCGG GTCAAT $-3{ }^{\prime}$.

\section{CRISPR/Cas9 knock out (KO) cell lines}

SMMC-7721 cells were transfected with FBXL6 CRISPR/Cas9 KO (h) KO plasmid (sc-408,853, Santa Cruz Biotechnology) using Lipofectamine2000 following the manufacturer's instructions. Cells were selected with $1 \mu \mathrm{g} / \mathrm{ml}$ puromycin 2 weeks. Single clones were then selected and the knockout efficiency was verified by western blot assay. 


\section{Western blotting and antibodies}

Cells were lysed with lysis buffer $(100 \mathrm{mM}$ Tris- $\mathrm{HCl}, \mathrm{pH}$ 6.8, $100 \mathrm{mM}$ DTT, 1\% SDS, $10 \%$ glycerol). Proteins were separated by $10-12 \%$ SDS-PAGE, and transferred to NC membrane. Membranes were blocked in 5\% non-fat milk in phosphate-buffered saline (PBS) for $1 \mathrm{~h}$ before incubation with primary antibody overnight at $4{ }^{\circ} \mathrm{C}$. Membranes were washed with and incubated with secondary antibody for $1 \mathrm{~h}$. Primary antibodies used as indicated: anti-Flag M2 (1:4000 dilution, F1804, Sigma), antiHSP90AA1 (1:1000 dilution, 13,171-1-AP, Protein tech), anti-FBXL6 (1:1000 dilution, SAB1407299, Sigma), antiCul1 (1:1000 dilution, sc-17,775, Santa cruz, U.S.A), anti-SKP1 (1:2000 dilution, \#12248, Cell Signaling Technology, U.S.A), anti-c-Myc (1:1000 dilution, \#18583, Cell Signaling Technology, U.S.A), and antiGAPDH (1:5000 dilution, \#5174, Cell Signaling Technology, U.S.A).

\section{Immunoprecipitation (IP) and mass spectrometry (MS)}

Cells were lysed with IP buffer $(100 \mathrm{mM} \mathrm{NaCl}, 20 \mathrm{mM}$ Tris-cl PH8.0, 0.5 mM EDTA, 0.5\% (v/v) Nonidet P-40) with protease inhibitor cocktail and phosphorylate inhibitor for $30 \mathrm{~min}$ on ice. Cells were sonicated and the lysates were centrifuged. The supernatant was incubated with appropriate antibodies and protein A/G beads overnight at $4{ }^{\circ} \mathrm{C}$ in a rotating wheel. Immunoprecipitates were washed with IP buffer. SDS loading buffer was then added and proteins were eluted by boiling at $95^{\circ} \mathrm{Cfor} 5$ min. For mass spectrometry assay, lysates from $293 \mathrm{~T}$ cells transfected with Flag-con or Flag-FBXL6 were cleared by centrifugation at $15,000 \mathrm{~g}$ for $20 \mathrm{~min}$ at $4{ }^{\circ} \mathrm{C}$ to remove cell debris. The resulting lysates were subjected to IP with Flag M2 beads overnight at $4{ }^{\circ} \mathrm{C}$. Bound proteins were eluted by boiling, resolved by SDS-PAGE and stained with coomassie blue staining, followed by mass spectrometry analysis.

\section{In vivo Ubiquitination assay}

Cells co-transfected His-K63-Ubiquitin with EV or FlagFBXL6 plasmids were sonicated in IP buffer containing $8 \mathrm{M}$ urea and $10 \mathrm{mM}$ imidazole. His-K63-Ubiquitin-conjugated proteins were recovered with Ni-NTA resin (Qiagen), washed eight times in urea lysis buffer containing $20 \mathrm{mM}$ imidazole, and eluted with IP buffer containing 5\% SDS and $200 \mathrm{mM}$ imidazole. The boiled samples were separated by $10 \%$ SDS-PAGE and subjected to western blot with antibodies as indicated. For endogenous ubiquitinated protein accumulation, Tandem Ubiquitin Binding Entity 2 (TUBE2) resin (LifeSensors) was used. Cells were lysed with IP buffer with protease inhibitor cocktail and phosphorylate inhibitor for $30 \mathrm{~min}$ on ice. Cells were sonicated and the lysates were centrifuged. The supernatant was incubated with TUBE2 resin overnight at $4{ }^{\circ} \mathrm{C}$ in a rotating wheel. The resin was then washed with IP buffer and boiled in SDS loading buffer. Boiled samples were separated by $10 \%$ SDS-PAGE and subjected to western blot with antibodies as indicated.

\section{Colony formation analysis}

Cells were seeded in a six-well plate at a density of $1000 /$ well and then cultured for 2 weeks. The numbers of colonies containing more than 50 cells were counted by crystal purple staining.

\section{Apoptosis analysis}

Cells were seeded into 6 well plates. Apoptosis cells were determined using Annexin V-fluorescein isothiocyanate (FITC) and propidium iodide (PI) apoptosis detection kit according to the manufacturer's instruction. Cell apoptosis was then analyzed using a FACS Calibur flow cytometer (BD Biosciences, San Jose, CA, USA). Apoptosis was also determined by measuring the activity of the caspases 3 and 7 using a luminescent substrate (Caspase-Glo 3/7; Promega) according to manufacturer's instructions.

\section{Luciferase reporter and chromatin immunoprecipitation assays}

The promoter region of FBXL6 gene was amplified from the human genomic DNA and inserted into pGL4.15 vector (Promega, Madison, Wisconsin, USA). For the luciferase reporter assays, HEK293T cells were seeded in 24-well plates and transfected with the indicated plasmids using Lipofectamine 2000 (Invitrogen) for $36 \mathrm{~h}$. Luciferase activity was measured using the Dual Luciferase Reporter Assay System (Promega). The firefly luciferase luminescence data were normalized by the Renilla luciferase luminescence data. A chromatin immunoprecipitation (ChIP) assay kit (Upstate, Billerica, MA) was used according to manufacturer instructions. Briefly, cells were fixed with formaldehyde and DNA was sheared to fragments at $200-1000$ bp by repeated sonication. Chromatin was then incubated and precipitated with antibodies against c-Myc or IgG. Primers for GAPDH were used as negative control.

\section{Xenograft assays}

Animal study was approved by Animal Care and Use Committee of Fudan University Shanghai Cancer Center. 8-week-old male BALB/cA nude mice were purchased from National Rodent Laboratory Animal Resources (Shanghai, China). All mice were kept in a specific pathogen-free facility and housed at $21^{\circ} \mathrm{C} \pm 1{ }^{\circ} \mathrm{C}$ with humidity of $55 \pm 10 \%$, fed with sterilized food and water, and kept on a $12 \mathrm{~h} \mathrm{light/dark}$ cycle. FBXL6 ${ }^{+/+}$and FBXL6 $^{-1-}$ SMMC-7721 cells at a density of $1 \times 10^{7}$ were suspended in $50 \mu \mathrm{l}$ of DMEM medium, mixed 1:1 with Matrigel (Corning) and injected into the flanks of male 
nude mice. Tumor sizes were measured by a caliper and calculated using the formula length $\times$ width $2 \times 1 / 2$. Tumor weights were measured after mice were sacrificed.

\section{Statistical analyses}

All experiments were at least repeated three times. Data are presented as mean \pm standard deviation (SD). Statistical analysis was performed with GraphPad Prism 7.0 software. The differences between groups were calculated using the Student's t-test or one-way ANOVA using a Tukey post-hoc test. $P$ values of $<0.05$ were considered statistically significant. Statistical significance is displayed as * $P<0.05, * P<0.01$, and ${ }^{* * * *} P<0.001$, respectively.

\section{Results}

\section{FBXL6 is highly expressed in HCC and associated with the} c-MYC pathway

To identify key genes involved in the tumorigenesis of HCC, transcriptome RNA-sequencing data of 374 primary $\mathrm{HCC}$ samples and 50 non-tumor tissues were downloaded from the TCGA data portal (https:// cancergenome.nih.gov/). The Limma $\mathrm{R}$ package identified 7667 differentially expressed genes, 7273 upregulated and 394 down-regulated (Fig. 1a-b). The output of the whole differentially expressed genes was provide in the supplementary Table 1 . Among those upregulated genes, we are particularly interested in F-box proteins, which are usually involved in the development of diverse cancers [23]. For example, the most famous Fbox proteins are SKP2, $\beta$-TrcP and FBXW7, which are known oncogenes or tumor suppressors [24-26]. We found that the mRNA levels of some F-box proteins were significantly increased in HCC samples when compared with non-tumor tissues, including FBXL18, FBXL16 and FBXL6. FBXL18 has been reported to play an oncogenic role in glioma through promoting K63linked ubiquitination of Akt [27]. However, the biological function of FBXL16 and FBXL6 proteins are poorly reported. It has been reported that FBXL16 could not interact with Cullin1 to form a SCF complex, indicating an E3 ligase independent function of FBXL16 [28]. Thus, in the current study, we focused on FBXL6, an orphan F-box protein, the expression of which was significantly increased in $\operatorname{HCC}(P=2.75 \mathrm{E}-25)$ (Fig. 1c).

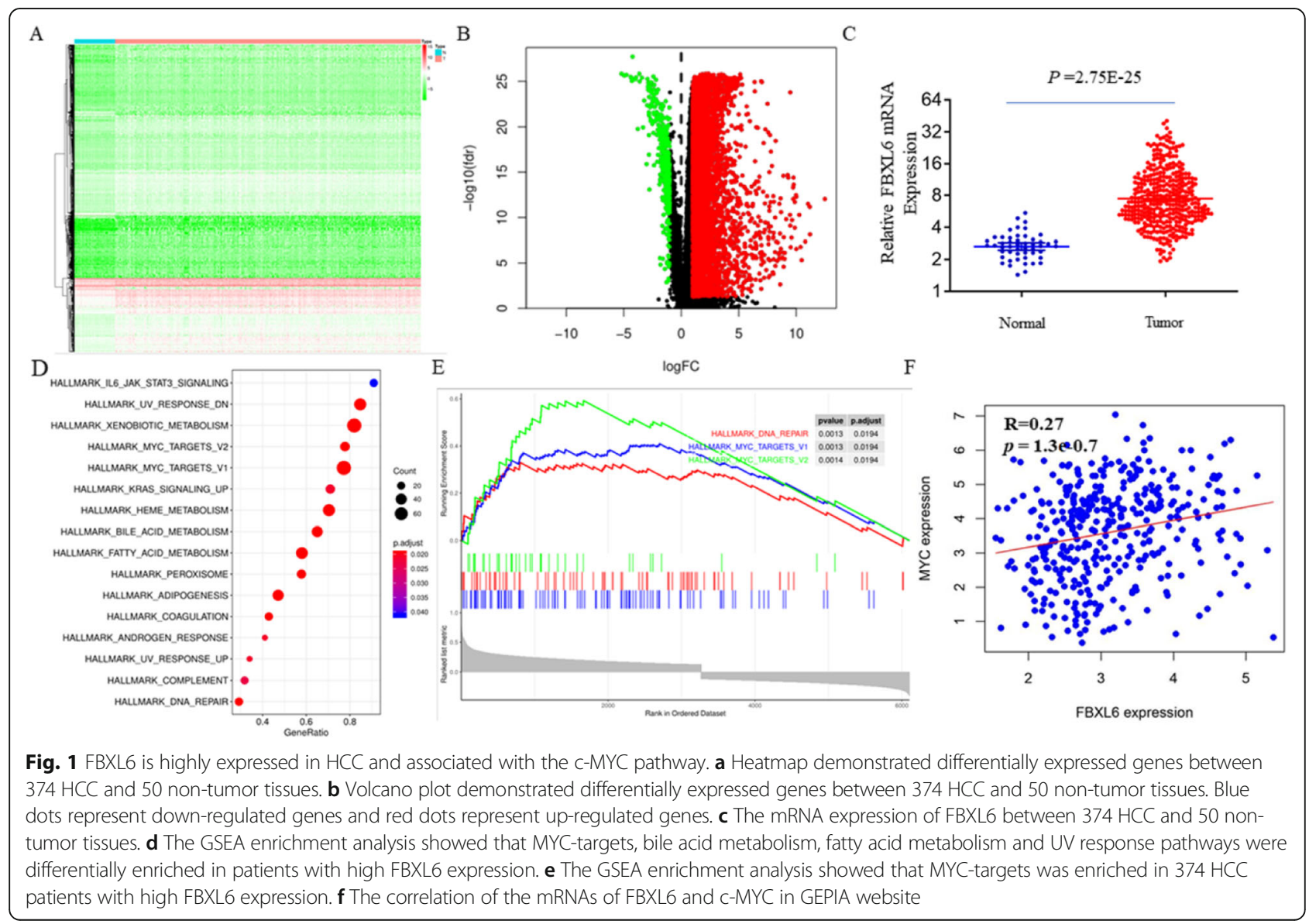


In $374 \mathrm{HCC}$ samples, the expression correlation coefficients of FBXL6 and all other genes were calculated using R (Supplementary Table S2), and the Gene Set Enrichment Analysis (GSEA) enrichment analysis was performed using the GSEABase package. We identified many pathways that were significantly enriched, such as MYC-targets, bile acid metabolism, fatty acid metabolism and UV response (Fig. 1d), suggesting that FBXL6 might play a role in these pathways. Notably, given the critical role of c-MYC oncogene in the tumorigenesis of HCC, the enrichment of MYC-target signature suggested a potential regulation of FBXL6 by c-MYC in HCC (Fig. 1e, Supplementary Figure 1). In supporting with this notion, we found that the c-MYC and FBXL6 mRNAs have a notable correlation in liver cancer samples $(R=0.27$, $P=1.3 \mathrm{e}-0.7$ ) (Fig. 1f) [29]. Moreover, the expression of FBXL6 was also correlated with many c-MYC target genes including 56.1\% MYC activating genes (73/130) and $41.9 \%$ MYC repressed genes (13/31) (Supplementary Table S2). Together, these data suggested FBXL6 was highly expressed in HCC samples and associated with the c-MYC pathway.

\section{FBXL6 exhibits tumor-promoting ability in HCC}

To examine the roles of FBXL6 in growth control, we firstly used two small hairpin RNA (shRNA) constructs to reduce the expression of FBXL6 in Hep3B cells (Fig. 2a). Silencing the expression of FBXL6 caused delayed cell growth and reduced colony formation ability (Fig. 2b-c). Flow cytometry assay showed that the spontaneous apoptosis rate of FBXL6-depelted Hep3B cells was higher than that of control cells (Fig. 2d), and the activities of caspase 3 and caspase7 were also enhanced in the absence of FBXL6 (Fig. 2e). To avoid selection of HCC lines that may not accurately reflect the effects of FBXL6 deletion, we also constructed FBXL6 knock out

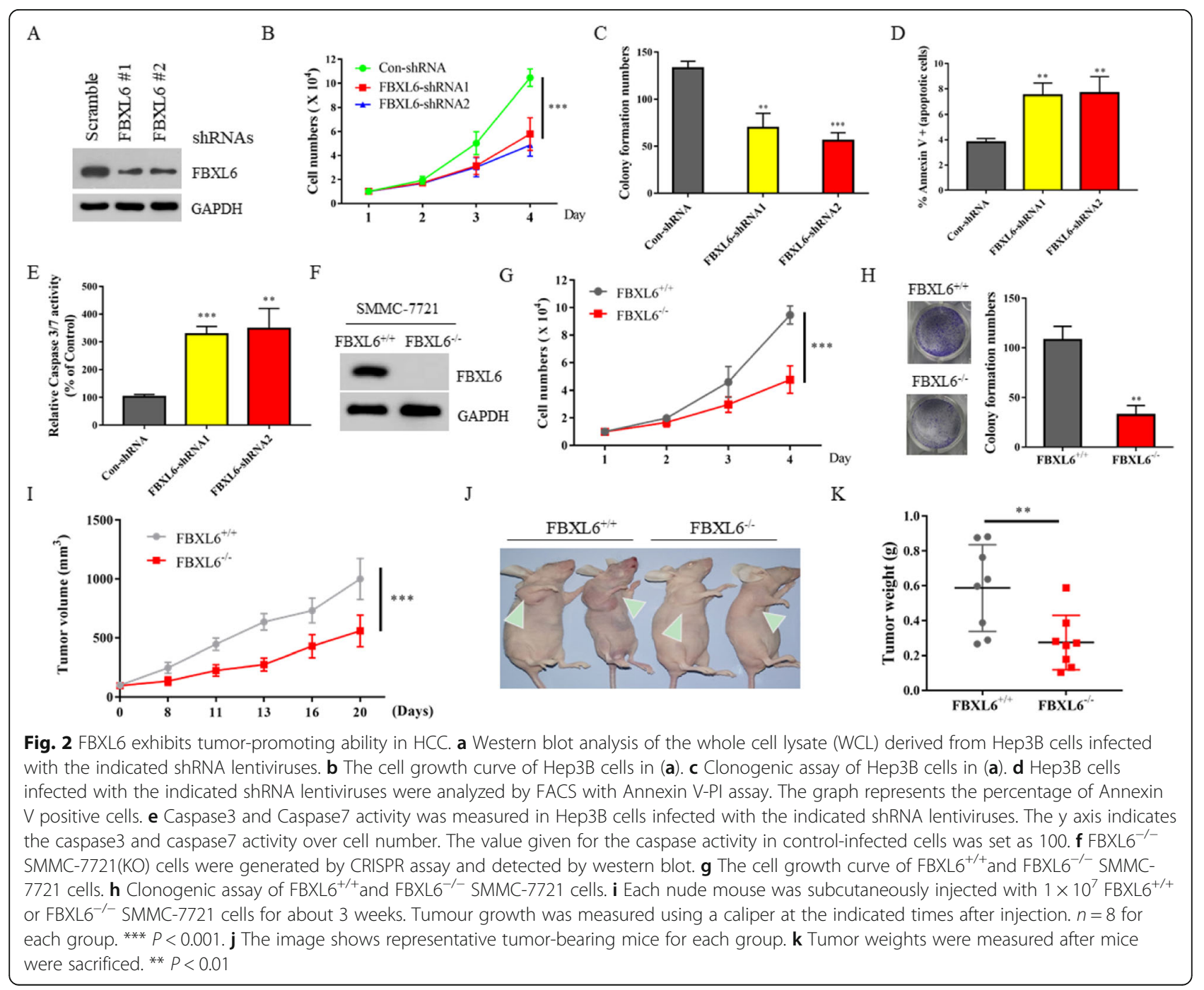


(KO) SMMC-7721 cell line using CRISPR-Cas9 technology, and found that FBXL6 deficiency decreased proliferation and colony formation compared with the control wild type (WT) cells (Fig. 2f-h). Furthermore, we also used nude mice model to investigate whether FBXL6 affected HCC cells proliferation in vivo. Four weeks old $\mathrm{BALB} / \mathrm{c}$ nude mice were subcutaneously injected with $1 \times 10^{7}$ WT or FBXL6 KO SMMC-7721 cells. We found that knock out of FBXL6 significantly decreased tumor volume and tumor weight compared with WT cells (Fig. 2i-k). Thus, these data indicated that FBXL6 played a critical role in liver cancer cells proliferation both in vitro and in vivo.

\section{HSP90AA1 is associated with FBXL6}

To investigate the molecule mechanism underline the tumor-promoting role of FBXL6, we used the immunoprecipitation/Mass Spectrum (IP/MS) method to identify the interacting proteins of FBXL6. Flag-FBXL6 or Flag-
Con plasmids were transfected into $293 \mathrm{~T}$ cells and the cell lysates of these cells were subjected to MS analysis after purification by Flag M2 beads. As expected, our MS data analysis identified the known FBXL6 interacting proteins such as Cullin1 and SKP1, suggesting FBXL6 forms a classical SCF E3 ligase complex with both proteins. Importantly, we also identified several unknown new interacting proteins such as HSP90AA1 (Fig. 3a). We then performed western blot assay to confirm our MS data. We found that Cullin1, SKP1 and HSP90AA1 could only be detected in Flag-FBXL6 immunoprecipitate (Fig. 3b). To demonstrate the specificity of this binding, we screened 9 human F-box protein family members. Flag-tagged F-box proteins were expressed into $293 \mathrm{~T}$ cells and then immunoprecipitated to evaluate their interaction with endogenous HSP90AA1 protein. Although each F-box protein binds to SKP1, the only F-box protein that binds to HSP90AA1 is FBXL6 (Fig. 3c). The endogenous interaction between FBXL6

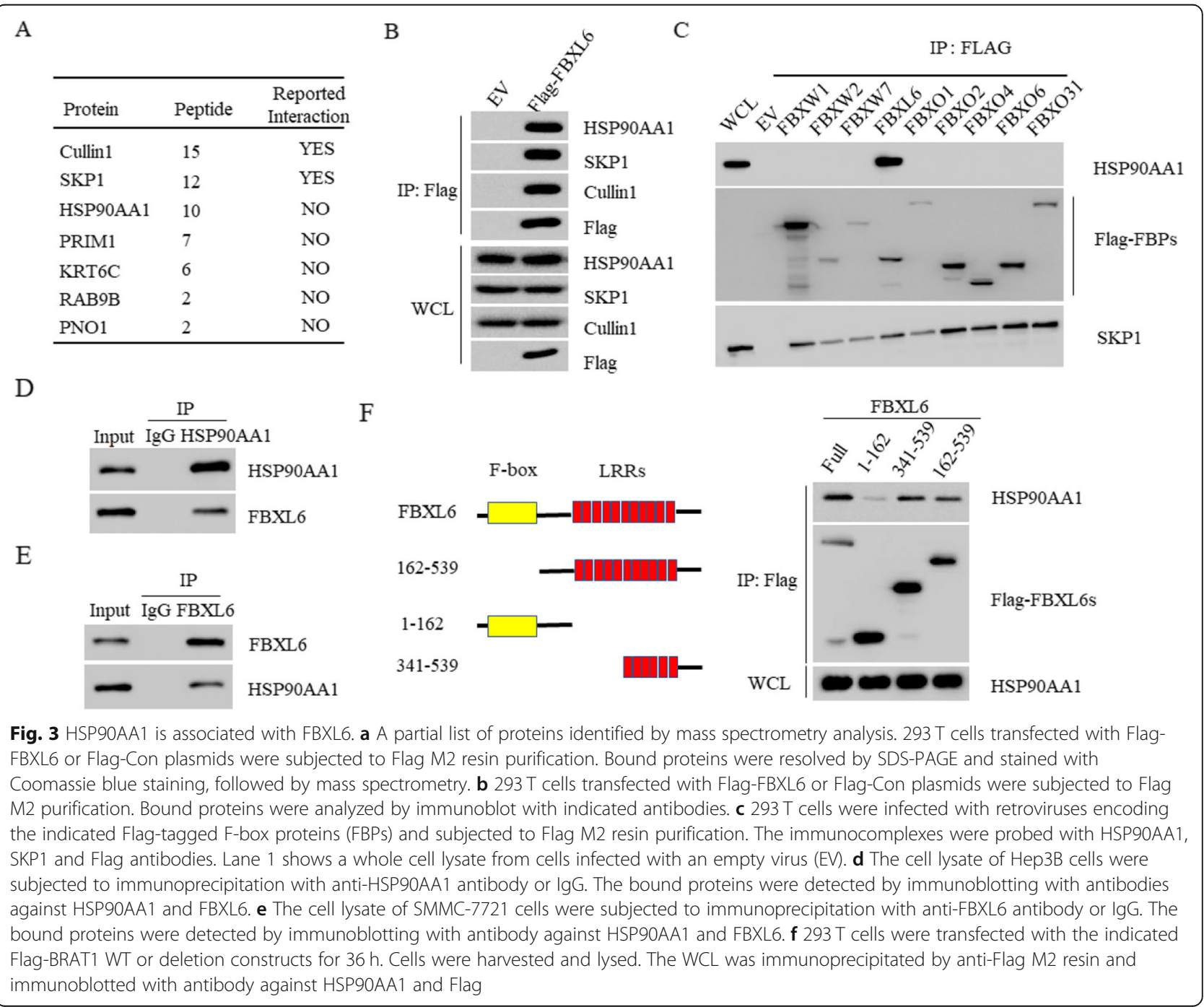


and HSP90AA1 was also verified in both Hep3B and SMMC-7721 cells (Fig. 3d-e). FBXL6 is composed of an $\mathrm{N}$-terminal F-box domain and multiple leucine-rich repeat sequences. By protein interaction domain mapping assay, we found that FBXL6 bound to HSP90AA1 via its leucine-rich repeat sequences at its C-terminus (Fig. 3f). Taken together, these data indicated that FBXL6 specifically interacted with HSP90AA1.

\section{FBXL6 stabilizes HSP90AA1 protein by promoting its K63-} ubiquitination

Typically, F-box proteins usually ubiquitinate substrate proteins and promote their proteasomal degradation [30]. In order to investigate whether FBXL6 can promote the degradation of HSP90AA1, we first overexpressed FBXL6 into SMMC-7721 cells. Interestingly, overexpression of FBXL6 did not reduce HSP90AA1 expression. Instead, it significantly induced the expression of HSP90AA1 protein, without affecting its mRNA level (Fig. 4a). The similar phenomenon was also observed in Hep3B cells (Fig. 4b). On the contrary, silencing the expression of FBXL6 by shRNAs significantly reduced HSP90AA1 expression (Fig. 4c). In agreement, the protein level, but not the mRNA level, of HSP90AA1 was significantly decreased in FBXL6 KO cells compared with WT cells (Fig. 4d). Furthermore, overexpression of an F-box domain-deleted FBXL6 (FBXL6 $\Delta$ F-box) mutant failed to regulate HSP90AA1 expression (Fig. 4e), suggesting that FBXL6-induced HSP90AA1expression required its E3 ligase activity. To test this possibility, we compared the half-life of HSP90AA1 in HCC cells with or without FBXL6 expression and found that the halflife of HSP90AA1 in FBXL6 KO cells was significantly reduced relative to the WT counterpart (Fig. 4f). Consistently, treatment with the protease inhibitor MG132 restored HSP90AA1 expression in FBXL6 KO cells. (Fig. $4 \mathrm{~g})$. However, overexpression of FBXL6 increased the global ubiquitination form of HSP90AA1, suggesting that FBXL6 might promote HSP90AA1 ubiquitination to prevent its degradation (Fig. 4h). Indeed, co-transfected with a His-ubiquitin-K63 plasmid, which coding ubiquitin with only K63 and other lysines were mutated to arginines, showed that FBXL6 significantly promoted HSP90AA1 K63-dependent ubiquitination (Fig. 4i). Since K63-dependent ubiquitination modifications usually do not participate in protein degradation, our data suggest that FBXL6 may promote K63-dependent ubiquitination of HSP90AA1 to stabilize it. In addition, we conducted an IHC analysis to evaluate the potential association between FBXL6 and HSP90AA1 protein in 87 human HCC specimens using anti-FBXL6 and antiHSP90AA1 antibodies (Fig. 4j). We found that FBXL6 was positively correlated with HSP90AA1 protein in these samples (Fig. $4 \mathrm{k}, \mathrm{X}^{2}=19.24, P<0.001$ ).

\section{FBXL6 stabilizes c-MYC via HSP90AA1}

HSP90AA1 can exert its tumor-promoting effect by stabilizing c-MYC protein in several cancer types [22, 31]. We found that overexpression of HSP90AA1 in SMMC7721 and Hep3B also up-regulated c-MYC protein, suggesting c-MYC might be a critical client protein of HSP90AA1 in HCC (Fig. 5a). Moreover, overexpression of FBXL6 promoted the expression of c-MYC in a dosedependent manner in Hep3B cells (Fig. 5b). However, overexpression of FBXL6 $\Delta$ F-box mutant could not affect c-MYC expression, suggesting this regulation required the E3 ligase activity of FBXL6 (Fig. 5c). Consistent with this, the expression of c-MYC in FBXL6 KO cells was significantly reduced (Fig. 5d), the half-life of c-MYC protein was shortened (Fig. 5e), and the ubiquitinated form was increased (Fig. 5f). Unsurprisingly, there is no evidence to support the direct binding between FBXL6 and c-MYC protein, suggesting that FBXL6 may regulate c-MYC indirectly. Indeed, overexpression of HSP90AA1 in FBXL6 KO cells partially reversed the FBXL6-induced c-MYC expression (Fig. 5g). Therefore, these data indicated that FBXL6 stabilized $\mathrm{c}-\mathrm{MYC}$ protein via HSP90AA1.

\section{C-MYC transcriptional activates FBXL6 in HCC}

Bioinformatics analysis reveals that c-MYC and FBXL6 mRNAs have significant correlation in HCC samples (Fig. 1d). Since FBXL6 is an ubiquitin E3 ligase and cMYC is a classical transcription factor, we hypothesized that C-MYC may regulate the transcription of FBXL6 in HCC. Indeed, overexpression of c-MYC induced the mRNA expression of FBXL6 in both SMMC-7721 and Hep3B cells (Fig. 6a). On the contrast, knockdown of cMYC by siRNAs inhibited the mRNA expression of FBXL6 (Fig. 6b). The $\mathrm{C}$ terminus of c-MYC contains a HLH-LZ domain, which is known to bind to the canonical E-box (CACGTG) to regulate downstream genes expression [32]. We next performed Chromatin immunoprecipitation (ChIP) experiments in Hep3B cells to determine whether c-MYC directly binds to the genomic locus of FBXL6. $3 \mathrm{~KB}$ of sequence of the FBXL6 promoter region was then examined for putative c-MYCbinding sites. We used the Jaspar website to locate the exact position of the E box in the promoter of FBXL6 (http://jaspar.genereg.net/). The $-3000 \mathrm{bp}$ region from the TSS of FBXL6 was used to scan the potential binding sites of c-MYC. The top one sequence of the software provided was CACGTG, started at 551 and ended at 556 (input $3000 \mathrm{bp}$ ), and the score of which was 10.53. Thus, we identified one potential E-box in the FBXL6 promoter region (Fig. 6c). ChIP assay revealed that anti-c-MYC antibody efficiently immunoprecipitated $-2500 \mathrm{bp}$ to $-2300 \mathrm{bp}$ upstream from the transcription start site (TSS) of FBXL6 gene in Hep3B cells 


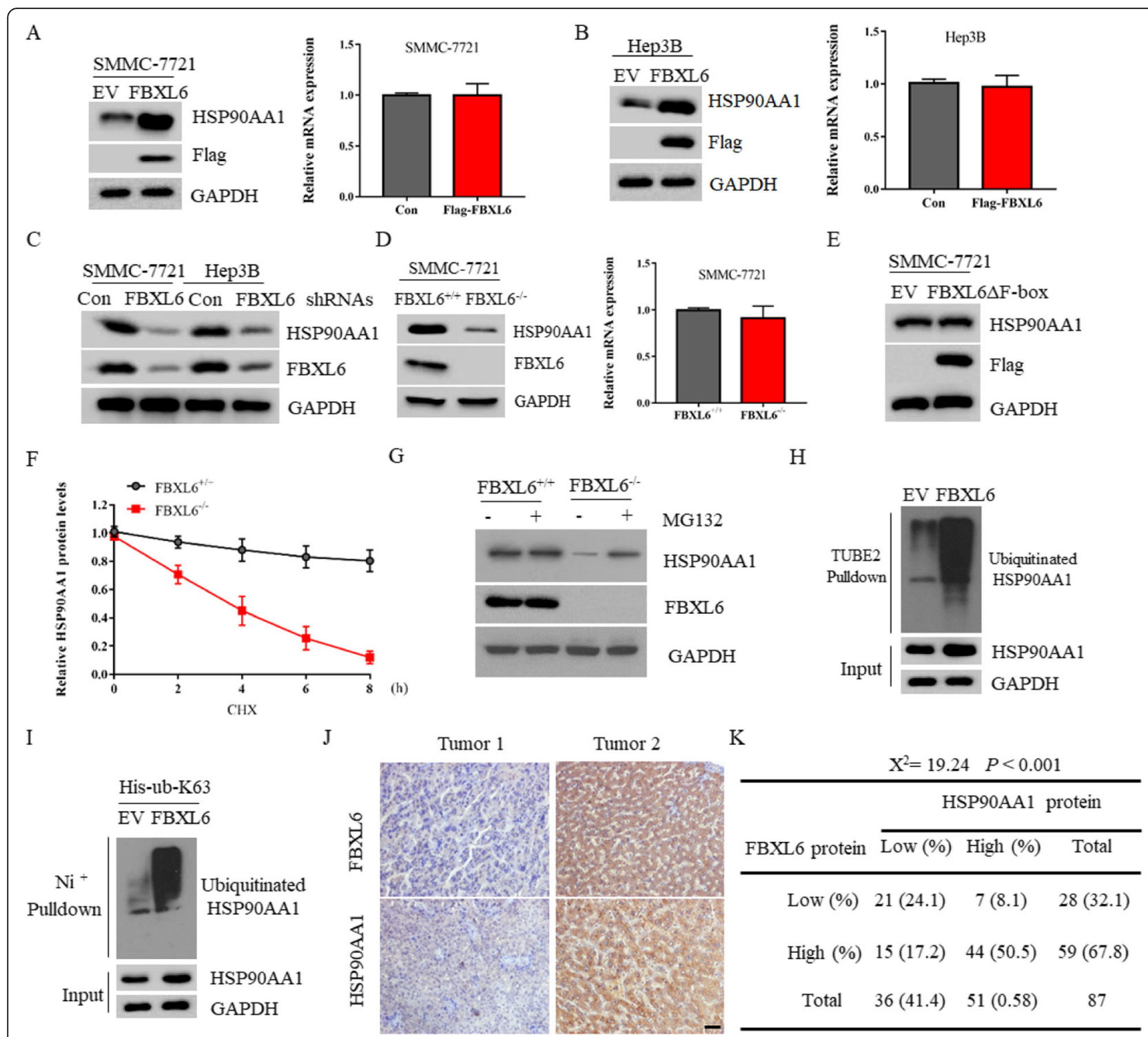

Fig. 4 FBXL6 stabilizes HSP90AA1 protein by promoting its K63-ubiquitination. a The protein and mRNA levels of HSP90AA1 from SMMC-7721 cells transfected with Flag-Con (EV) or Flag-FBXL6 indicated plasmids were detected by immunoblotting and real-time quantitative PCR, respectively. $\mathbf{b}$ The protein and mRNA levels of HSP90AA1 from Hep3B cells transfected with indicated plasmids were detected by immunoblotting and real-time quantitative PCR, respectively. c Western blot analysis of the WCL derived from SMMC-7721 cells or Hep3B cells infected with the indicated shRNA lentiviruses. $\mathbf{d}$ The protein and mRNA levels of HSP90AA1 from FBXL6 ${ }^{+/+}$and FBXL6 ${ }^{-/-}$SMMC-7721 cells were detected by immunoblotting and real-time quantitative PCR, respectively. e Western blot analysis of the WCL derived from SMMC-7721 cells transfected with EV or Flag-FBXL6 $\mathrm{F}$-box plasmids. $\mathrm{f} F B X L 6^{+/+}$and FBXL6 ${ }^{-/-}$SMMC-7721 cells were treated with $20 \mu \mathrm{M}$ cycloheximide (CHX) for the indicated time. The whole cell lysate was immunoblotted with anti-HSP90AA1 antibody. The quantification plot was based on scanning densitometry analysis using the Image I software. Relative protein levels were normalized to FBXL6 ${ }^{-/-}$control group. $\mathbf{g}$ Western blot analysis of the WCL derived from FBXL6 ${ }^{+/+}$and FBXL6 ${ }^{-/-}$SMMC-7721 cells treated with $10 \mu \mathrm{M}$ MG132 for 6 h. h SMMC-7721 cells transfected with EV or Flag-FBXL6 plasmids were harvested and lysed. WCL were immunoprecipitated by Tandem Ubiquitin Binding Entity 2 (TUBE2) resin for ubiquitinated proteins enrichment and immunoblotted as indicated. i SMMC-7721 cells were co-transfected Flag-FBXL6 and His-K63-ub for 36 h, cell lysate was subjected to immunoprecipitation by $\mathrm{Ni}^{+}$purification. Immunoprecipitate was detected by immunoblotting using the indicated antibodies. $\mathbf{j} \| \mathrm{HC}$ analysis was performed on 87 specimens from HCC patients using anti-FBXL6 and anti-HSP90AA1 antibodies. Representative images of HC staining of tumors of two HCC patients are presented. $\mathbf{k}$ The correlation study of FBXL6 and HSP90AA1 protein expression in 87 HCC samples is shown

(Fig. 6d), suggesting c-MYC directly binds to the promoter region of FBXL6. We also constructed a luciferase reporter vector containing the FBXL6 promoter region of this E-box. The luciferase reporter assay found that c-
MYC increased the promoter activity in cells transfected with E-box WT vectors but not in cells with E-box mutant vectors (Fig. 6e). Therefore, our data indicate that FBXL6 is a downstream target gene of c-MYC. 


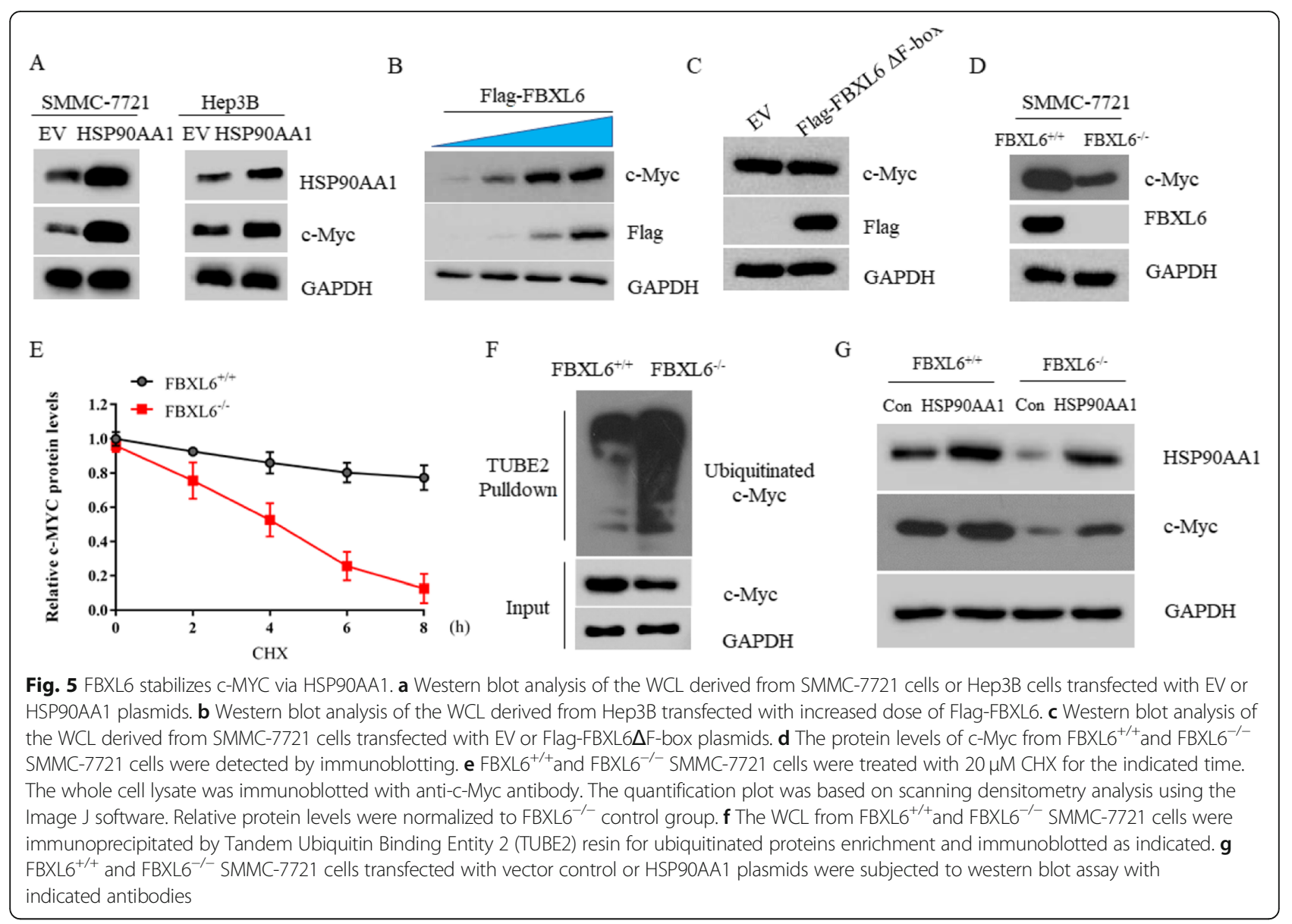

\section{Discussion}

Here, we report that FBXL6 stabilizes HSP90AA1 expression in HCC cells and that FBXL6 expression is correlated with c-MYC expression in HCC tissues, of which a great ratio expresses higher mRNA levels of FBXL6. In line with these observations, we show that genetic inhibition of FBXL6 eliminates HCC cells proliferation in vitro, and thus tumor progression in subcutaneously transplanted HCC mice, indicating a critical role of FBXL6 in the pathogenesis of HCC.

By using IP/MS assay, HSP90AA1 was identified as a putative FBXL6-interacting protein. We further showed that FBXL6 directly interacted with HSP90AA1 via its C-terminus leucine-rich repeat sequences. During the preparation of this manuscript, another group also found that HSP90AA1 was an interacting protein of FBXL6 by high throughput assay [33]. However, the relationship between FBXL6 and HSP90AA1 was still undetermined. Interestingly, unlike the canonical degradationpromoting function of most F-box proteins, we found that FBXL6 ubiquitinates HSP90AA1 to counteract its degradation. Our data further indicate that FBXL6 promotes K63-dependent ubiquitination of HSP90AA1, although we cannot rule out the possibility that FBXL6 may also stimulate other types of ubiquitination, which may help explain this discrepancy. It is possible that FBXL6-mediated HSP90AA1 K63-dependent ubiquitination may antagonize HSP90AA1 K48-dependent ubiquitination promoted by other E3 ligases. Indeed, another F-box protein, FBXL21, has been shown to ubiquitinate cryptochromes to stabilize these proteins [34, 35], suggesting the shared common underline mechanisms of FBXL6 and FBXL21 However, whether FBXL21 also promotes K63-dependent ubiquitination of cryptochromes is still unknown. Therefore, further research is still needed to understand the detailed mechanism of how FBXL6 stabilizes HSP90AA1 protein. The correlation expression of the mRNA levels of FBXL6 and cMYC promotes us to determine whether FBXL6 is transcriptional regulated by c-MYC. By using ChIP and luciferase assays, we found that $\mathrm{c}-\mathrm{MYC}$ directly bound to the E-box of FBXL6 promoter region to promote its mRNA expression, suggesting FBXL6 is a downstream target gene of $\mathrm{c}-\mathrm{MYC}$ in $\mathrm{HCC}$. 


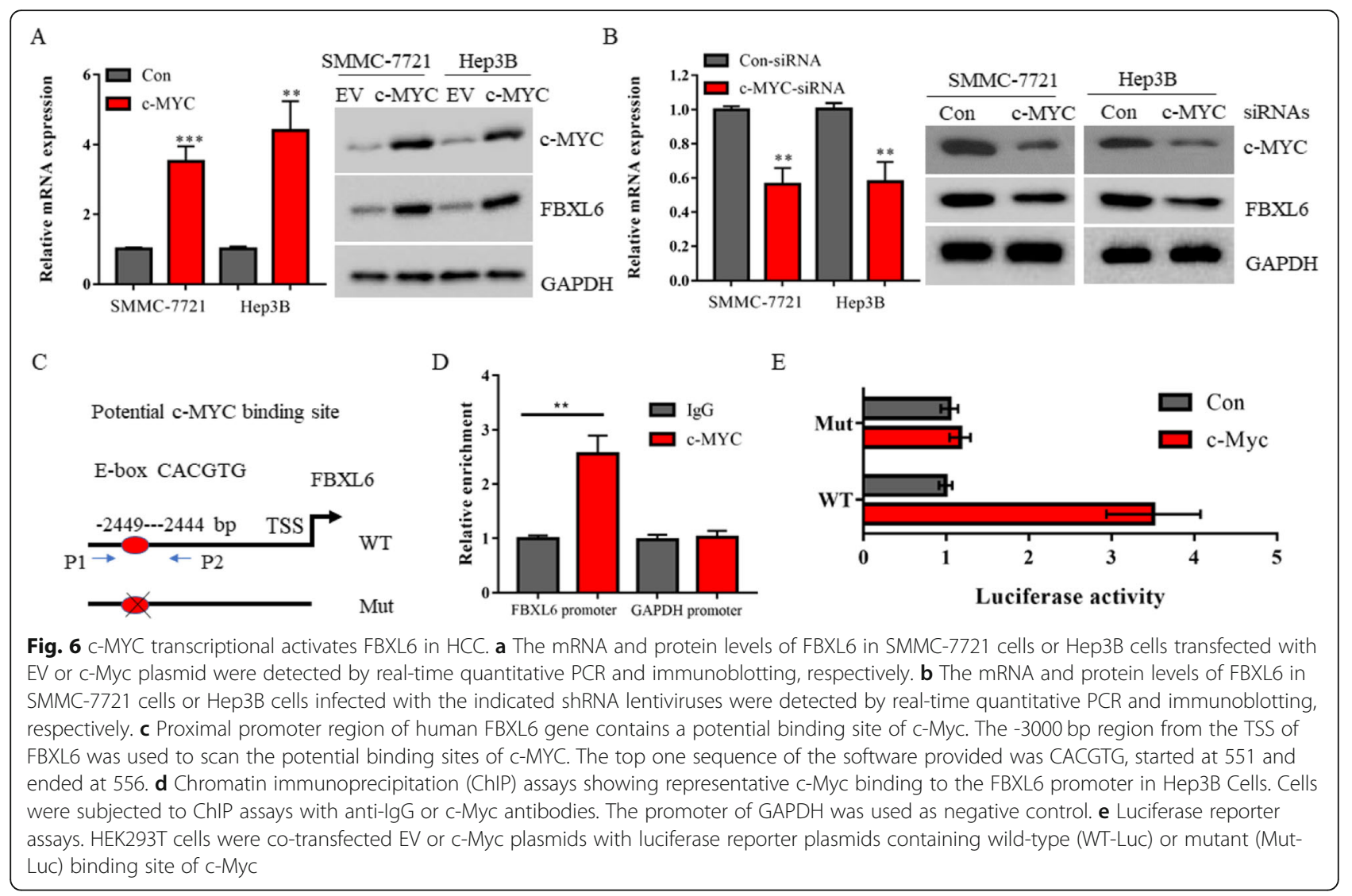

\section{Conclusions}

Therefore, our data reveals an unknown positive feedback loop of FBXL6-HSP90AA1-c-MYC axis, and its abnormal regulation may contribute to the occurrence of HCC, and suggests that agents targeting FBXL6 will be beneficial to inhibit HCC.

\section{Supplementary information}

Supplementary information accompanies this paper at https://doi.org/10. 1186/s12964-020-00604-y.

Additional file 1: Supplementary table 1. The output of all differentially expressed genes.

Additional file 2: Supplementary table 2. The correlations between FBXL6 mRNA expression and MYC target genes.

Additional file 3: Supplementary Figure 1. GSEA enrichment analysis of FBXL6 by utilizing the standard GSEA 4.0.1 software.

\section{Abbreviations}

APC: Anaphase-promoting complex; CRLs: Cullin-based ubiquitin E3 ligases; HCC: Hepatocellular carcinoma; HECT: Homologous to the E6AP carboxyl terminus; HSPs: Heat shock proteins; IP: Immunoprecipitation; LIHC: Liver hepatocellular carcinoma; Limma RING: Linear Models for Microarray and RNA-Seq Data, really interesting new gene; SCF: Skp1/Cullin1/F-box protein; UPS: Ubiquitin (Ub)-proteasome system

\section{Acknowledgements}

Not applicable.
Authors' contributions

WS and ZM collaborated to design the study. WS, LF, SD, ZN, YH and LL were responsible for experiments. WS, ZC and ZM analyzed the data. WS and ZM wrote the paper. All authors collaborated to interpret results and develop the manuscript. All authors read and approved the final version of the manuscript.

\section{Funding}

This study was supported by the National Science Foundation of China (81673746).

Availability of data and materials

Please contact corresponding author for data requests.

Ethics approval and consent to participate

Animal study was approved by Animal Care and Use Committee of Fudan University Shanghai Cancer Center.

Consent for publication

All listed authors have actively participated in the study and have read and approved the submitted manuscript.

\section{Competing interests}

The authors declare that they have no competing interests.

\section{Author details}

'Department of Integrative Oncology, Fudan University Shanghai Cancer Center, 270 Dong An Road, Shanghai 200032, China. 'Department of

Oncology, Shanghai Medical College, Fudan University, Shanghai, China.

${ }^{3}$ Collaborative Innovation Center for Cancer Medicine, Fudan University

Shanghai Cancer Center, Shanghai, China. 
Received: 28 December 2019 Accepted: 29 May 2020

Published online: 23 June 2020

\section{References}

1. Marquardt JU, Andersen JB, Thorgeirsson SS. Functional and genetic deconstruction of the cellular origin in liver cancer. Nat Rev Cancer. 2015;15: 653-67.

2. Sia D, Villanueva A, Friedman SL, Llovet JM. Liver Cancer cell of origin, molecular class, and effects on patient prognosis. Gastroenterology. 2017: 152:745-61

3. Reinstein $\mathrm{E}$, Ciechanover A. Narrative review: protein degradation and human diseases: the ubiquitin connection. Ann Intern Med. 2006;145:676-84.

4. Ciechanover A. The unravelling of the ubiquitin system. Nat Rev Mol Cell Biol. 2015;16:322-4.

5. Wickliffe KE, Williamson A, Meyer HJ, Kelly A, Rape M. K11-linked ubiquitin chains as novel regulators of cell division. Trends Cell Biol. 2011;21:656-63.

6. Branigan E, Plechanovova A, Jaffray EG, Naismith JH, Hay RT. Structural basis for the RING-catalyzed synthesis of K63-linked ubiquitin chains. Nat Struct Mol Biol. 2015;22:597-602.

7. Deshaies RJ, Joazeiro CA. RING domain E3 ubiquitin ligases. Annu Rev Biochem. 2009;78:399-434.

8. Nakayama Kl, Nakayama K. Ubiquitin ligases: cell-cycle control and cancer. Nat Rev Cancer. 2006;6:369-81

9. Jin J, Cardozo T, Lovering RC, Elledge SJ, Pagano M, Harper JW. Systematic analysis and nomenclature of mammalian F-box proteins. Genes Dev. 2004; 18:2573-80.

10. Bai C, Sen P, Hofmann K, Ma L, Goebl M, Harper JW, Elledge SJ. SKP1 connects cell cycle regulators to the ubiquitin proteolysis machinery through a novel motif, the F-box. Cell. 1996;86:263-74.

11. Wei W, Ayad NG, Wan Y, Zhang GJ, Kirschner MW, Kaelin WG Jr. Degradation of the SCF component Skp2 in cell-cycle phase G1 by the anaphase-promoting complex. Nature. 2004;428:194-8.

12. Bashir T, Dorrello NV, Amador V, Guardavaccaro D, Pagano M. Control of the SCF (Skp2-Cks1) ubiquitin ligase by the APC/C (Cdh1) ubiquitin ligase Nature. 2004:428:190-3.

13. Chan CH, Li CF, Yang WL, Gao Y, Lee SW, Feng Z, Huang HY, Tsai KK, Flores LG, Shao Y, et al. The Skp2-SCF E3 ligase regulates Akt ubiquitination, glycolysis, herceptin sensitivity, and tumorigenesis. Cell. 2012;149:1098-111.

14. Meng X, Liu X, Guo X, Jiang $S$, Chen T, Hu Z, Liu H, Bai Y, Xue M, Hu R, et al. FBXO38 mediates PD-1 ubiquitination and regulates anti-tumour immunity of T cells. Nature. 2018;564:130-5.

15. Wu J, Liu T, Rios Z, Mei Q, Lin X, Cao S. Heat shock proteins and Cancer. Trends Pharmacol Sci. 2017:38:226-56.

16. Feder ME, Hofmann GE. Heat-shock proteins, molecular chaperones, and the stress response: evolutionary and ecological physiology. Annu Rev Physiol. 1999;61:243-82.

17. Rutherford SL, Lindquist S. Hsp90 as a capacitor for morphological evolution. Nature 1998:396:336-42.

18. Mayer MP, Le Breton L. Hsp90: breaking the symmetry. Mol Cell. 2015;58:8-20.

19. Kreusch A, Han S, Brinker A, Zhou V, Choi HS, He Y, Lesley SA, Caldwell J, Gu XJ. Crystal structures of human HSP90alpha-complexed with dihydroxyphenylpyrazoles. Bioorg Med Chem Lett. 2005;15:1475-8.

20. Khandelwal A, Crowley VM, Blagg BSJ. Natural product inspired N-terminal Hsp90 inhibitors: from bench to bedside? Med Res Rev. 2016:36:92-118.

21. Xu Q, Tu J, Dou C, Zhang J, Yang L, Liu X, Lei K, Liu Z, Wang Y, Li L, et al. HSP90 promotes cell glycolysis, proliferation and inhibits apoptosis by regulating PKM2 abundance via Thr-328 phosphorylation in hepatocellular carcinoma. Mol Cancer. 2017;16:178.

22. Regan PL, Jacobs J, Wang G, Torres J, Edo R, Friedmann J, Tang XX. Hsp90 inhibition increases p53 expression and destabilizes MYCN and MYC in neuroblastoma. Int J Oncol. 2011;38:105-12.

23. Wang Z, Liu P, Inuzuka H, Wei W. Roles of F-box proteins in cancer. Nat Rev Cancer. 2014;14:233-47.

24. Lin HK, Chen Z, Wang G, Nardella C, Lee SW, Chan CH, Yang WL, Wang J, Egia A, Nakayama Kl, et al. Skp2 targeting suppresses tumorigenesis by Arfp53-independent cellular senescence. Nature. 2010;464:374-9.

25. Westbrook TF, Hu G, Ang XL, Mulligan P, Pavlova NN, Liang A, Leng Y, Maehr R, Shi Y, Harper JW, Elledge SJ. SCFbeta-TRCP controls oncogenic transformation and neural differentiation through REST degradation. Nature. 2008:452:370-4.
26. Mao JH, Perez-Losada J, Wu D, Delrosario R, Tsunematsu R, Nakayama Kl, Brown K, Bryson S, Balmain A. Fbxw7/Cdc4 is a p53-dependent haploinsufficient tumour suppressor gene. Nature. 2004;432:775-9.

27. Zhang J, Yang Z, Ou J, Xia X, Zhi F, Cui J. The F-box protein FBXL18 promotes glioma progression by promoting K63-linked ubiquitination of Akt. FEBS Lett. 2017:591:145-54.

28. Honarpour N, Rose CM, Brumbaugh J, Anderson J, Graham RL, Sweredoski MJ, Hess S, Coon JJ, Deshaies RJ. F-box protein FBXL16 binds PP2AB55alpha and regulates differentiation of embryonic stem cells along the FLK1+ lineage. Mol Cell Proteomics. 2014;13:780-91.

29. Tang Z, Li C, Kang B, Gao G, Li C, Zhang Z. GEPIA: a web server for cancer and normal gene expression profiling and interactive analyses. Nucleic Acids Res. 2017:45:W98-W102.

30. Skaar JR, Pagan JK, Pagano M. Mechanisms and function of substrate recruitment by F-box proteins. Nat Rev Mol Cell Biol. 2013;14:369-81.

31. Paul I, Ahmed SF, Bhowmik A, Deb S, Ghosh MK. The ubiquitin ligase CHIP regulates c-Myc stability and transcriptional activity. Oncogene. 2013;32: 1284-95.

32. Zeller Kl, Jegga AG, Aronow BJ, O'Donnell KA, Dang CV. An integrated database of genes responsive to the Myc oncogenic transcription factor: identification of direct genomic targets. Genome Biol. 2003:4:R69.

33. Chen D, Liu X, Xia T, Tekcham DS, Wang W, Chen H, Li T, Lu C, Ning Z, Liu $X$, et al. A Multidimensional Characterization of E3 Ubiquitin Ligase and Substrate Interaction Network. iScience. 2019:16:177-91.

34. Hirano A, Yumimoto K, Tsunematsu R, Matsumoto M, Oyama M, KozukaHata H, Nakagawa T, Lanjakornsiripan D, Nakayama Kl, Fukada Y. FBXL21 regulates oscillation of the circadian clock through ubiquitination and stabilization of cryptochromes. Cell. 2013;152:1106-18.

35. Yoo SH, Mohawk JA, Siepka SM, Shan Y, Huh SK, Hong HK, Kornblum I, Kumar V, Koike N, Xu M, et al. Competing E3 ubiquitin ligases govern circadian periodicity by degradation of CRY in nucleus and cytoplasm. Cell. 2013;152:1091-105.

\section{Publisher's Note}

Springer Nature remains neutral with regard to jurisdictional claims in published maps and institutional affiliations.

Ready to submit your research? Choose BMC and benefit from:

- fast, convenient online submission

- thorough peer review by experienced researchers in your field

- rapid publication on acceptance

- support for research data, including large and complex data types

- gold Open Access which fosters wider collaboration and increased citations

- maximum visibility for your research: over $100 \mathrm{M}$ website views per year

At BMC, research is always in progress.

Learn more biomedcentral.com/submissions 